\title{
New Parameters for Evaluating Cerebral Autoregulation and Pressure-Volume Compensatory Reserve in Neurocritial Patients
}

\author{
Won-Sang $\mathrm{Cho}^{1}$, Eun Jin $\mathrm{Ha}^{1,4}$, Sang Bae $\mathrm{Ko}^{2,4}$, Seungman Yang ${ }^{3,5}$, Hee Chan Kim ${ }^{3,5}$, Jeong Eun Kim ${ }^{1}$ \\ ${ }^{1}$ Department of Neurosurgery, Seoul National University Hospital, Seoul National University College of Medicine, Seoul, Korea \\ ${ }^{2}$ Department of Neurology, Seoul National University Hospital, Seoul National University College of Medicine, Seoul, Korea \\ ${ }^{3}$ Department of Biomedical Engineering, Seoul National University Hospital, Seoul National University College of Medicine, Seoul, Korea \\ ${ }^{4}$ Critical Care Center, Seoul National University Hospital, Seoul, Korea \\ ${ }^{5}$ Interdisciplinary Program in Bioengineering, Seoul National University, Seoul, Korea
}

Received: September 11, 2018

Accepted: September 19, 2018

Published: October 10, 2018

\section{Corresponding Author:}

Won-Sang Cho, M.D., Ph.D.

Associate Professor Department of

Neurosurgery, Seoul National

University Hospital, 101 Daehak-

ro, Seoul 03080, Korea

Tel: +82-2-2072-2824

Fax: +82-2-744-8459

E-mail: nsdrcho@gmail.com
The goals of neurocritical care are to resolve primary causes, minimize secondary insults and maximize patient recovery. Concepts such as intracranial pressure (ICP), cerebral perfusion pressure (CPP) and cerebral blood flow have been long applied in neurocritical care; however, patient care involving these concepts alone is not satisfactory. Recently, new developments have allowed integrative physiological and biochemical data to be acquired and analyzed using multimodality monitoring with invasive devices. In addition, newly developed parameters have contributed to improvement in clinical outcomes in neurocritical patients. Beyond the indiscriminate application of threshold values for ICP and CPP in all patients, precision care can be provided by finding the optimal values of parameters and adjusting treatment for each patient in a particular time period. Here, we introduce representative parameters including the pressure reactivity index $(\mathrm{PRx})$ for evaluating the status of cerebral autoregulation and optimal CPP $\left(\mathrm{CPP}_{\text {opt }}\right)$ and the correlation between the amplitude of ICP and the mean ICP (RAP) for assessing the pressure-volume compensatory reserve. Additionally, we present a bedside software and monitoring system that we recently developed for analyzing these parameters.

Keywords: Neurocritical care; PRx; $\mathrm{CPP}_{\text {opp }}$ RAP

\section{INTRODUCTION}

Practice and knowledge in the field of neurocritical care have rapidly progressed. Physicians have managed neurocritical patients for a long time based on methods with a low level of evidence and their experience. However, neurointensivists currently care for patients by integrating methods with a higher level of evidence, new devices and parameters and by determining optimal values of physiological and biochemical parameters in each individual patient. We tried to introduce significant parameters for the pressure reactivity index (PRx) and the correlation between the pulse amplitude of intracranial pressure (ICP) and the mean ICP (RAP) with a new bedside monitoring system that they developed recently. 


\section{HISTORICAL PERSPECTIVE}

Neurocritical care seems to have begun from the time point when the concept of ICP was formed. The existence of ICP was first recognized by Alexander Monro approximately 200 years ago. The Monro-Kellie hypothesis or doctrine suggests that the brain, cerebrospinal fluid (CSF) and blood maintain the balance of ICP within a nonexpandable skull ${ }^{1)}$. When a space-occupying lesion develops, CSF and venous blood decrease, and ICP is controlled within the normal range of pressure. However, when the volume of a new lesion is beyond the compensatory capacity, ICP abruptly increases followed by a decrease in cerebral perfusion pressure (CPP) and cerebral blood flow (CBF) and ultimately brain herniation. Harvey Cushing developed the modern field of neurocritical care. He reported a research paper on the association among ICP, systemic arterial pressure and respiration in $1901^{2}$. Saline-induced increases in ICP corresponds to increases in systemic arterial blood pressure and respiratory depression. Based on the previous research, the 'Cushing reflex' was defined. In the 1960s, Nil Lundberg began directly measuring ICP by inserting a ventricular catheter and reported normal and pathologic waveforms of $\mathrm{ICP}^{3)}$. Thereafter, ICP measurement was applied in the clinical setting for treating neurocritical patients. ICP control is most important goal of neurocritical care, especially in patients with traumatic brain injury. There are many guidelines for the care of neurocritical patients, among which the guidelines from the Brain Trauma Foundation are the most well described in detail ${ }^{4}$. The Brain Trauma Foundation published the first edition of "Guidelines for the management of severe traumatic brain injury" in 1995, and successive editions have been updated in 1999, 2007 and 2016. Among them, a detailed description of each topic was reported in the $3^{\text {rd }}$ version of the guidelines in 2007. The $3^{\text {rd }}$ version dealt with blood pressure and oxygenation, hyperosmolar therapy, prophylactic hypothermia, infection prophylaxis, deep vein thrombosis prophylaxis, indications for ICP monitoring, ICP monitoring technology, ICP thresholds, CPP thresholds, brain oxygen monitoring and thresholds, anesthetics, analgesics and sedatives, nutrition, antiseizure prophylaxis, hyperventilation and steroids. In the chapters about ICP and CPP thresholds, treatment with a low level of evidence is recommended to maintain ICP below $20 \mathrm{mmHg}$ and CPP between 50 and 70 $\mathrm{mmHg}$. These thresholds were not updated even in the $4^{\text {th }}$ edition of the guidelines published in 2016. In addition, those are recommended in patients with intracerebral hemorrhage ${ }^{5)}$ and acute ischemic stroke ${ }^{6}$ as they are. The fixed thresholds for ICP and CPP are currently applied like a central dogma in the genetics.

However, physicians have felt that these thresholds are inappropriate in some patients and insufficient in improving clinical outcomes. Recently, in 2012, a randomized controlled trial was conducted in South America that compared the survival rates of patients with traumatic brain injury between an ICP monitoring group (maintaining ICP below $20 \mathrm{mmHg}$ ) and another group of patients who were monitored with imaging and clinical examinations ${ }^{7}$. The study failed to show the superiority of ICP monitoring compared with the other group. Some questions still exist including the followings: (1) Are ICP and CPP sufficient for saving the brain? (2) Are the thresholds for ICP and CPP truly absolute for all patients? (3) Are ICP values that are measured truly exact measurements?

From these questions regarding the usefulness of ICP and CPP values, new physiological and biochemical parameters have been developed. In addition to ICP, CPP and CBF, data regarding brain oxygenation, glucose metabolism, neuronal electrical activity, brain water content, etc., are simultaneously collected and used for evaluating brain conditions and modulating appropriate treatment in each patient in a technique called 'multimodality monitoring ${ }^{8)}$. Multimodality monitoring is applied in limited medical institutions all over the world; however, this technique is expected to become popular in Korea in the near future.

\section{CEREBRAL AUTOREGULATION AND ITS MEASUREMENT}

The existence of cerebral autoregulation was established by Danish physicians in the $1970 \mathrm{~s}^{9}$. Cerebral autoregulation works between a mean arterial blood pressure (MAP) of 50 and 150 $\mathrm{mmHg}$, and CBF is steadily maintained within the range of 45$50 \mathrm{~mL} / 100 \mathrm{~g} / \mathrm{min}$ by automatic regulation of vessel diameters. Thereafter, in 1997, a Cambridge group of physicians reported a new parameter that was able to indirectly measure the functional status of cerebral autoregulation, namely, the "pressure reactivity index $(\mathrm{PRx})^{\left.{ }^{10}\right)}$. The PRx is the correlation coefficient between MAP and ICP. When cerebral autoregulation is impaired, increases in ICP lead to subsequent increases in MAP and cerebral blood volume in order to maintain $\mathrm{CBF}$, and a vicious cycle without any regulations can form. In this situation, MAP and ICP are positively proportional, and the PRx increases. When cerebral autoregulation is preserved, increases in ICP are buffered by vasoconstriction and outflow of CSF and venous blood, resulting in limited increases in MAP. Thus, the slope between MAP and ICP becomes smoother, and the PRx decreases (Fig. 1). In real clinical situations, a group with lower $\mathrm{PRx}$ values showed more favorable outcomes than a group with higher values ${ }^{10)}$. Although there are some differences in standard $\mathrm{PRx}$ values among reports, $\mathrm{PRx}$ values higher than $0.2-0.3$ are generally considered to indicate impaired cerebral autoregulation. 


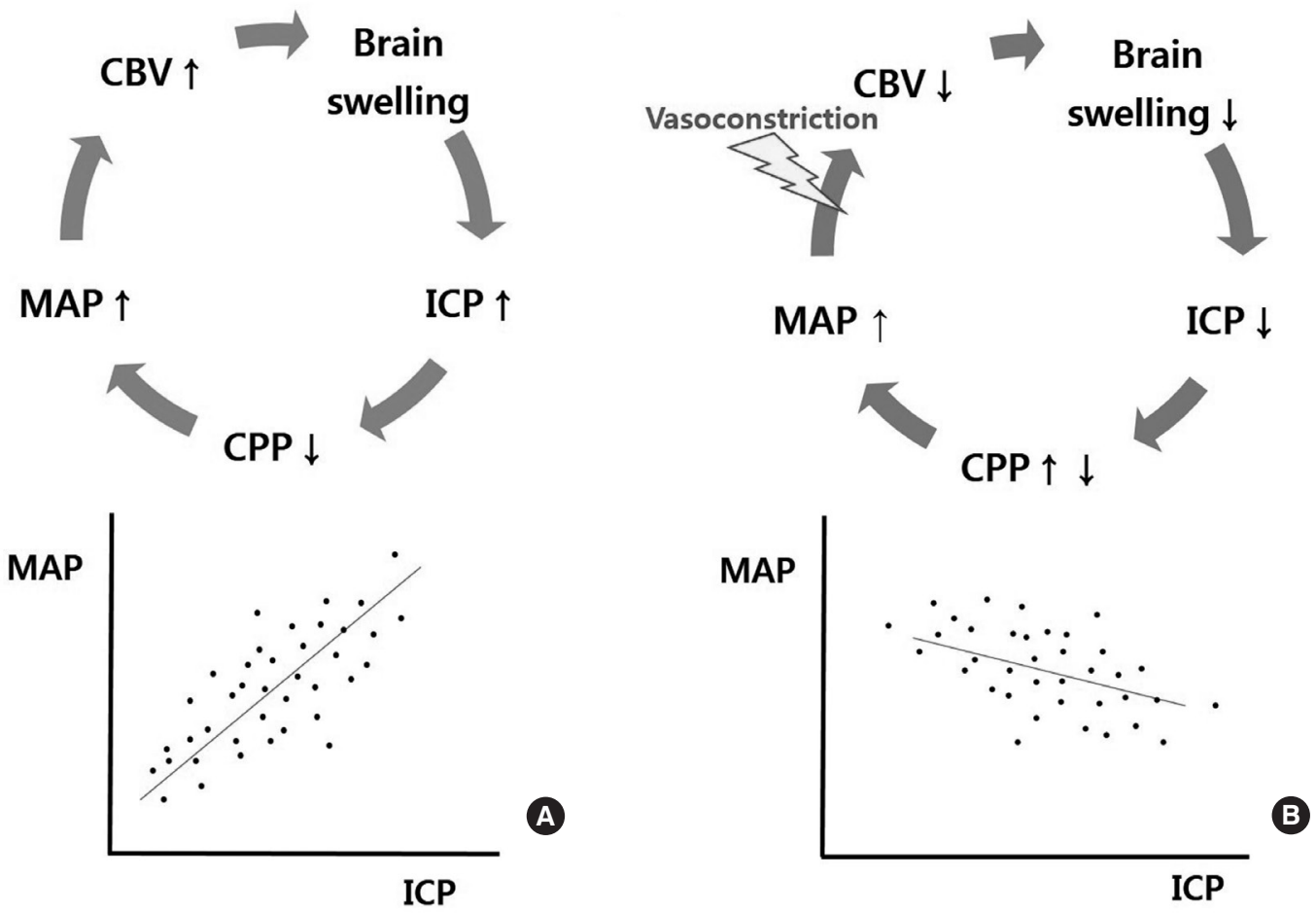

Fig. 1. Concept and definition of the pressure reactivity index $(\mathrm{PRx})$. A: In patients with impaired cerebral autoregulation, a vicious cycle occurs (upper), and the correlation coefficient (PRx) between MAP and ICP is positively increased (lower). B: In patients with preserved cerebral autoregulation, a positive cycle is formed by cerebral autoregulation (upper), and the PRx decreases and sometimes becomes negative (lower) (This is modified from reference 10). CBV, cerebral blood volume; MAP, mean arterial pressure; CPP, cerebral perfusion pressure; ICP, intracranial pressure.

In contrast, PRx values lower than $0.2-0.3$ are thought to indicate preserved autoregulation.

The same group subsequently proposed the concept of optimal $\mathrm{CPP}\left(\mathrm{CPP}_{\mathrm{opt}}\right)$ in $2002^{11)}$. When the PRx is at the lowest value, cerebral autoregulation is considered to be at its best, and CPP at this PRx value is also the best. Actually, when CPP is controlled as close to $\mathrm{CPP}_{\text {opt }}$ as possible, clinical outcomes are better. Thus, the most appropriate $\mathrm{CPP}$ is not within the absolute range of $\mathrm{CPP}=50-70 \mathrm{mmHg}$, as recommended in some guidelines, and the best CPP can be different under different conditions in each patient (Fig. 2). Some institutions have developed bedside software and monitoring systems for acquiring real time PRx and $\mathrm{CPP}_{\text {opt }}$ measurements. Among them, the ICM+ software ${ }^{\oplus}$ (Cambridge, UK) and monitoring system from the Cambridge group is the most popular. Recently, we also developed a prototype of a Korean version of a software monitoring system.

\section{PRESSURE-VOLUME COMPENSATORY RESERVE AND RAP}

The Cambridge group also demonstrated a new parameter

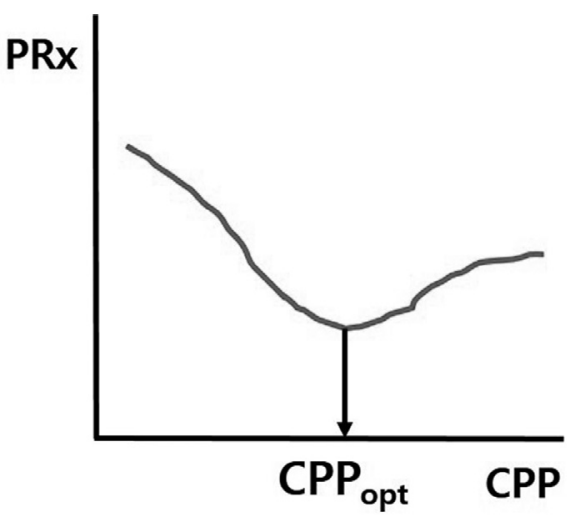

Fig. 2. Concept of $\mathrm{CPP}_{\text {opt }}$. When the PRx is the lowest, cerebral autoregulatory function is considered to be the best, and CPP at the lowest PRx is defined as $\mathrm{CPP}_{\mathrm{opt}}$ (This is modified from reference 11). CPP, cerebral perfusion pressure; $\mathrm{CPP}_{\text {opt }}$ optimal CPP; PRx, pressure reactivity index.

for evaluating the pressure-volume compensatory reserve in $2004^{12)}$. After gathering ICP data for a fixed time interval and distributing the ICP waveforms according to the frequency, waveforms are divided into 3 components: slow waves less than 
$0.1 \mathrm{~Hz}$, respiratory waves between 0.2 and $0.3 \mathrm{~Hz}$, and pulse waves higher than $1 \mathrm{~Hz}$ generated by heart rate. Among them, the first pulse wave with the highest amplitude is detected at approximately $1 \mathrm{~Hz}$, and the pulse amplitude of ICP (AMP) is defined as the amplitude of the first pulse wave.

Then, the correlation coefficient between AMP and mean ICP is defined as RAP. When the value of RAP is closer to 0 , the pressure-volume compensatory reserve is considered to be good; when the value of RAP becomes closer to 1 , the pressurevolume compensatory reserve is considered to be poor; and when the value of RAP becomes negative, brain function is thought to have shut down (Fig. 3). As intracranial volume increases and ICP begins to increase beyond the reserve capacity, AMP subsequently increases, and RAP ultimately increases; however, when ICP is constantly maintained with the aid of the compensatory reserve, AMP decreases and the value of RAP becomes closer to 0. RAP and the PRx are thought to be useful parameters for evaluating brain condition and modulating the types and intensity of treatment. As mentioned above, a bedside monitoring system developed by our group can display both of these features (Fig. 4).

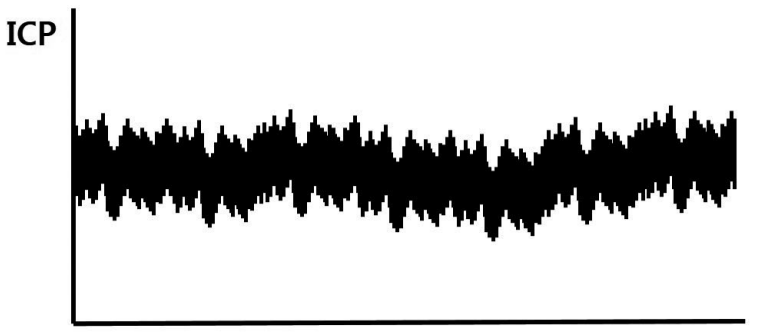

Time

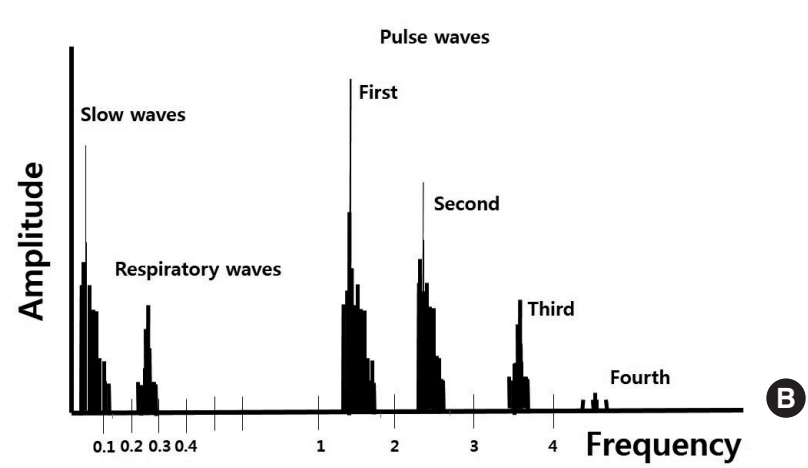

\section{CONCLUSION}

The era is declining in which neurocritical patients are managed based on methods with a low level of evidence and the uniform criteria of conventional parameters. Recently, neurocritical care is performed based on personalized data and new parameters obtained from multimodality monitoring. Representative parameters include the $\mathrm{PRx}, \mathrm{CPP}_{\mathrm{opt}}$, and RAP. As newly developed devices and data are applied to manage these patients, their final outcomes are expected to improve, and we can save more patients.

\section{CONFLICT OF INTEREST}

No potential conflict of interest relevant to this article was reported.

\section{ACKNOWLEDGEMENTS}

This research was supported grant (03-2017-0430) from the Seoul National University Hospital Research Fund.
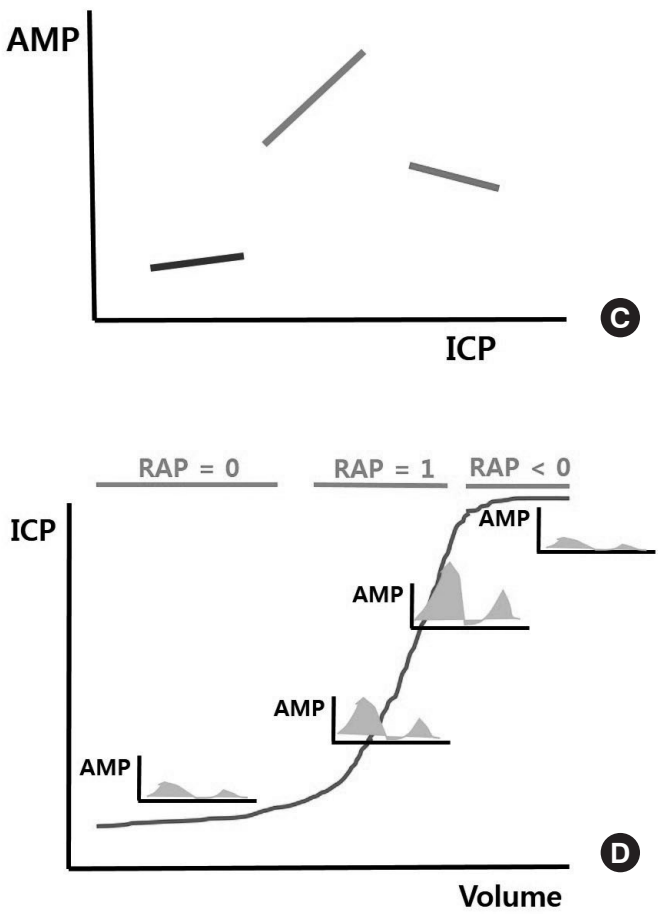

Fig. 3. Concept of RAP. A: ICP waveforms in a timeline. B: After unfolding the ICP waves within a fixed time interval, some wave peaks are grouped in different frequency sections. The amplitude of the first pulse wave at approximately $1 \mathrm{~Hz}$ is defined as AMP. C and D: RAP is the correlation coefficient between AMP and the mean ICP. As the pressure-volume compensatory reserve decreases, AMP increases, and the value of RAP consequently becomes closer to 1 . When the brain is completely damaged, AMP abruptly decreases, and the value of RAP can become negative (This is modified from reference 12). ICP, intracranial pressure; AMP, amplitude of ICP; RAP, correlation coefficient between AMP and mean ICP. 


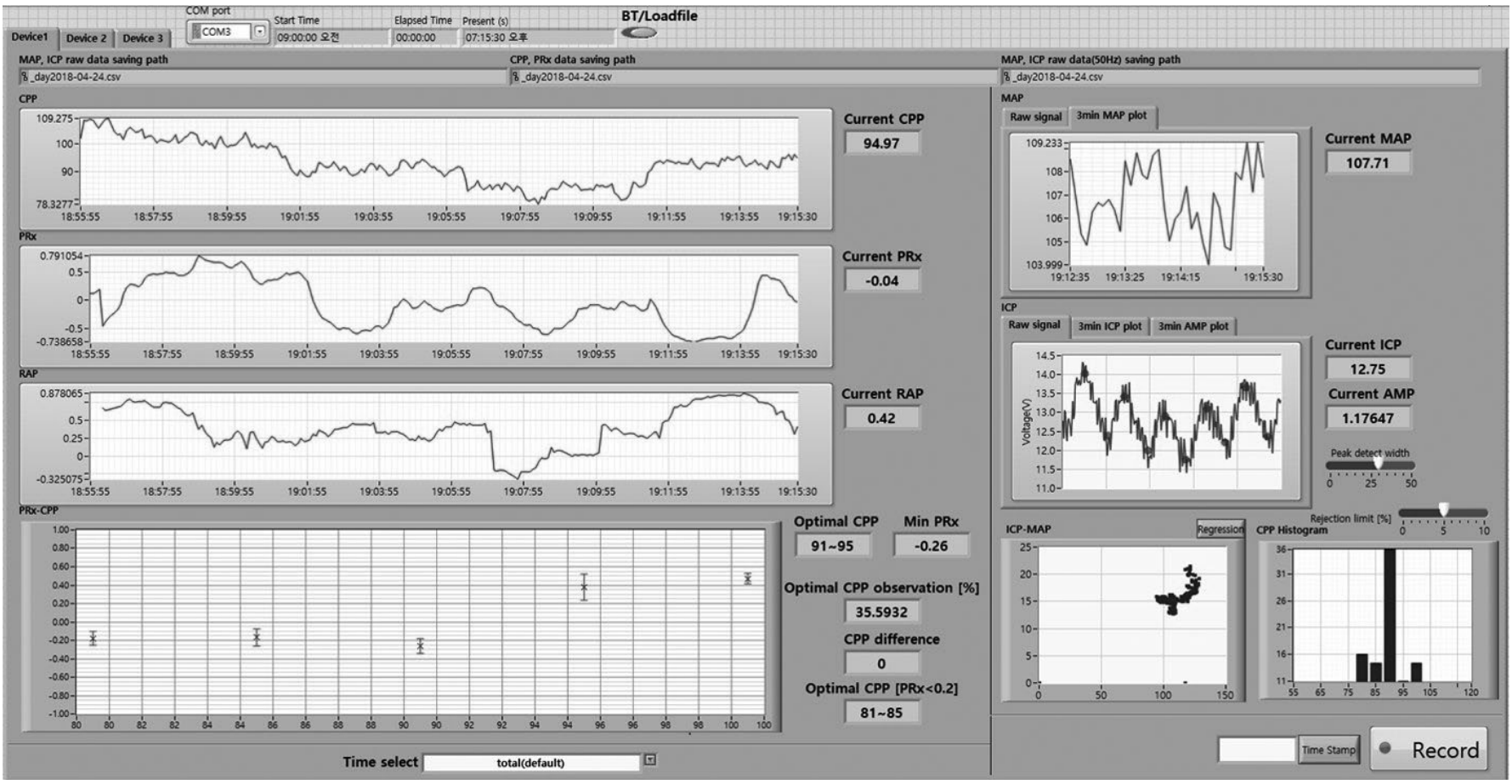

Fig. 4. New bedside software and monitoring system that we developed. This system displays ICP, MAP, CPP, PRx, CPP graphs and histograms. ICP, intracranial pressure; MAP, mean arterial pressure; CPP, cerebral perfusion pressure; $\mathrm{PRx}$, pressure reactivity index; $\mathrm{CPP}_{\mathrm{op}}$, optimal CPP; RAP, correlation coefficient between AMP and mean ICP.

\section{REFERENCES}

1. Andrews PJ, Citerio G. Intracranial pressure. Part one: historical overview and basic concepts. Intensive Care Med 2004;30:1730-1733.

2. Cushing H. Concerning a definite regulatory mechanism of the vasomotor center which controls blood pressure during cerebral compression. Bull Johns Hopkins Hosp $1901 ; 12: 290$.

3. Lundberg N. Continuous recording and control of ventricular fluid pressure in neurosurgical practice. Acta Psychiatr Scand Suppl 1960;36:1-193.

4. Brain Trauma Foundation. The 4th edition of guidelines for management of severe traumatic brain injury [Internet]. Brain trauma foundation; 2016 [cited 2018 July 16]. Available from: https://www.braintrauma.org.

5. Hemphill JC 3rd, Greenberg SM, Anderson CS, Becker K, Bendok BR, Cushman M, et al. Guidelines for the management of spontaneous intracerebral hemorrhage: a guideline for healthcare professionals from the american heart association/american stroke association. Stroke 2015;46:2032-2060.

6. Powers WJ, Rabinstein AA, Ackerson T, Adeoye OM, Bambakidis NC, Becker K, et al. 2018 guidelines for the early management of patients with acute ischemic stroke: a guideline for healthcare professionals from the american heart association/american stroke association. Stroke 2018;49:e46-e110.

7. Chesnut RM, Temkin N, Carney N, Dikmen S, Rondina C, Videtta W, et al. A trial of intracranial-pressure monitoring in traumatic brain injury. N Engl J Med 2012;367:2471-2481.

8. Ko SB. Multimodality monitoring in the neurointensive care unit: a special perspective for patients with stroke. J Stroke 2013;15:99-108.

9. Strandgaard S, Olesen J, Skinhoj E, Lassen NA. Autoregulation of brain circulation in severe arterial hypertension. Br Med J 1973;1:507-510.

10. Czosnyka M, Smielewski P, Kirkpatrick P, Laing RJ, Menon D, Pickard JD. Continuous assessment of the cerebral vasomotor reactivity in head injury. Neurosurgery 1997;41:11-17.

11. Steiner LA, Czosnyka M, Piechnik SK, Smielewski P, Chatfield D, Menon DK, et al. Continuous monitoring of cerebrovascular pressure reactivity allows determination of optimal cerebral perfusion pressure in patients with traumatic brain injury. Crit Care Med 2002;30:733-738.

12. Balestreri M, Czosnyka M, Steiner LA, Schmidt E, Smielewski P, Matta B, et al. Intracranial hypertension: what additional information can be derived from ICP waveform after head injury? Acta Neurochir (Wien) 2004;146:131141. 